\title{
Positioning system for sensor less linear DC motor.
}

\begin{abstract}
Linear motor has gain popularity as linear motion drive in office and factory automation which provides an alternative method to conventional rotary motor. Chip mounting is one of linear motor applications especially for linear DC motor (LDM). Displacement sensor which is normally used for positioning on the system, made the system expensive. This paper discusses the application of sensor less on positioning system for LDM. A spring is used to determine the position, while the spring displacement which is proportional to the thrust was produced by the motor. The thrust itself can be controlled by controlling the current supplied to the motor. Therefore, the positioning of LDM can be controlled by varying the current value. The approach used in this paper is to control the current is by manipulating various pattern of pulse width modulation (PWM) signal. As a result, the settling time less than $32 \mathrm{~ms}$ for $10 \mathrm{~mm}$ positioning has been achieved.
\end{abstract}

Keyword: Linear DC Motor; Spring Constant; PWM Signal; Duty Cycle; Quadraple Stage. 